\title{
Space-time compositional models: an introduction to simplicial partial differential operators
}

\author{
JARAUTA-BRAGULAT ${ }^{1}$, E. and EGOZCUE ${ }^{1}$, J. J. \\ ${ }^{1}$ Dept. Matemàtica Aplicada III - ETS Enginyers de Camins, Canals i Ports de Barcelona \\ Universitat Politècnica de Catalunya, Barcelona, Spain \\ (e-mail: eusebi.jarauta@upc.edu ; juan.jose.egozcue@upc.edu)
}

\begin{abstract}
A function assigning a composition to space-time points is called a compositional or simplicial field. These fields can be analysed using the compositional analysis tools. A study of linear models for evolutionary compositions depending on one variable, usually time, was formulated by Egozcue and Jarauta-Bragulat (2014) in terms of the so-called simplicial linear differential equations. The foundations of differential and integral calculus for simplex-valued functions of one real variable, was presented by Egozcue, Jarauta-Bragulat and Díaz-Barrero (2011).

In order to study compositions depending on space and/or time, reformulation and interpretation of traditional partial differential operators is required. These operators such as: partial derivatives, compositional gradient, directional derivative and divergence are of primary importance to state alternative models of processes as diffusion, advection and waves, from the compositional perspective. This kind of models, usually based on continuity of mass, circulation of a vector field along a curve and flux through surfaces, should be analyzed when compositional operators are used instead of the traditional gradient or divergence. This study is aimed at setting up the definitions, mathematical basis and interpretation of such operators.
\end{abstract}

\section{Introduction}

In a large number of processes studied in Sciences and in Engineering, magnitudes or variables involved can be modelled by a vector. This vector may be a function of one or several variables. Furthermore, in many cases the studied vector is a composition. Study of the evolutionary compositions depending on one variable was introduced by Egozcue and Jarauta-Bragulat (2014). The present work focuses on compositions whose evolution depends on several variables; in many cases, these variables are spatial coordinates and time. For example, the study of the evolution of a pollutant carried by a fluid stream.

For spatial coordinates, a subset $S \in \mathbb{R}^{d}$ of a $d$-dimensional real space is considered and identified with a physical domain; consequently $d=1, d=2$ and $d=3$ are the common choices of that dimension. A location in this space is denoted $s \in S$ and represented in a Cartesian coordinate system; for $d=1$ the spatial coordinate is usually denoted $s=x$; for $d=2, s=(x, y)$ and so on. For time, a subset $T \subseteq \mathbb{R}$ is considered and a point is denoted $t \in T$. For spatial and time evolutionary processes, a domain $S \times T \subseteq \mathbb{R}^{d} \times \mathbb{R} \quad(d=1,2,3)$ is considered.

A space-time vector-valued field with positive components $\mathbf{Z}$ is a function $\mathbf{Z}: S \times T \subseteq \mathbb{R}^{d} \times \mathbb{R} \rightarrow \mathbb{R}_{+}^{n}$ that assigns a positive component vector $\mathbf{Z}(s, t) \in \mathbb{R}_{+}^{n}$ to a space-time point $(s, t) \in S \times T$. If closure operation is then applied: $\mathcal{C} \mathbf{Z}(s, t)=\mathbf{z}(s, t)$ a space-time simplicial field $\mathbf{z}$ (STSF) is obtained. Consequently, a STSF is a function $\mathbf{z}: S \times T \subseteq \mathbb{R}^{d} \times \mathbb{R} \rightarrow \mathbb{S}^{n}$, that assigns a composition $\mathbf{z}(s, t)$ in the $n$-part simplex $\mathbb{S}^{n}$ to any space-time point $(s, t) \in S \times T$. In general, differentiability of $\mathbf{z}(s, t)$ up to the second order is assumed, thus guaranteeing the existence of continuous derivatives up to second order. 


\section{Derivatives and Integrals of a Space-Time Simplicial Field}

In the following, definitions and properties are developed for $d=2$ and extension to other values of $d$ are natural. Based on the definition of (ordinary) simplicial derivative (Egozcue et al., 2011) and the real calculus, natural definitions for partial simplicial derivatives of a STSF follow.

Definition 2.1 (SPatial And time simplicial DeRIVAtives) Let $\mathbf{z}: S \times T \subseteq \mathbb{R}^{2} \times \mathbb{R} \rightarrow \mathbb{S}^{n}$ be a $S T S F$ and $(x, y) \in S$. The spatial-partial simplicial derivatives of $\mathbf{z}$ are

$$
\begin{aligned}
& \partial_{x}^{\oplus} \mathbf{z}(x, y, t)=\lim _{h \rightarrow 0}\left(\frac{1}{h} \odot(\mathbf{z}(x+h, y, t) \ominus \mathbf{z}(x, y, t))\right), \\
& \partial_{y}^{\oplus} \mathbf{z}(x, y, t)=\lim _{h \rightarrow 0}\left(\frac{1}{h} \odot(\mathbf{z}(x, y+h, t) \ominus \mathbf{z}(x, y, t))\right) .
\end{aligned}
$$

The time-partial simplicial derivative is

$$
\partial_{t}^{\oplus} \mathbf{z}(x, y, t)=\lim _{h \rightarrow 0}\left(\frac{1}{h} \odot(\mathbf{z}(x, y, t+h) \ominus \mathbf{z}(x, y, t))\right) .
$$

Definition 2.2 (Directional simplicial Derivatives) Let $\mathbf{z}: S \times T \subseteq \mathbb{R}^{2} \times \mathbb{R} \rightarrow \mathbb{S}^{n}$ be $a$ STSF. Let $(x, y) \in S$ and $\vec{u}=\left(u_{x}, u_{y}\right)$ be a vector in $\mathbb{R}^{2}$. The simplicial derivative of $\mathbf{z}$ with respect to $\vec{u}$ is

$$
\partial_{\vec{u}}^{\oplus} \mathbf{z}(x, y, t)=\lim _{h \rightarrow 0}\left(\frac{1}{h} \odot\left(\mathbf{z}\left(x+h u_{x}, y+h u_{y}, t\right) \ominus \mathbf{z}(x, y, t)\right)\right) .
$$

If $\vec{u}$ is a unit vector, the derivative is called the directional simplicial derivative of $\mathbf{z}$.

Spatial-partial and time-partial simplicial derivatives are computed as they were ordinary simplicial derivatives of a single variable simplex-valued function as developed in Egozcue et al. (2011). Consequently, they can be computed as ordinary derivatives of the log-transformation of the STSF, and then transformed back into compositions. The same scheme works for clr and ilr transformations of $\mathbf{z}$ (Aitchison, 1986; Egozcue et al., 2003). The following proposition summarizes this kind of computation.

Proposition 2.1 Partial simplicial derivatives of $\mathbf{z}(x, y, t)$ can be computed as

$$
\partial_{x}^{\oplus} \mathbf{z}(x, y, t)=\mathcal{C} \exp \left(\partial_{x} \log (\mathbf{z}(x, y, t))\right)=\mathcal{C} \exp \left(\begin{array}{c}
\frac{\partial_{x} z_{1}(x, y, t)}{z_{1}(x, y, t)} \\
\vdots \\
\frac{\partial_{x} z_{n}(x, y, t)}{z_{n}(x, y, t)}
\end{array}\right) .
$$

Additionaly

$$
\operatorname{clr}\left(\partial_{x}^{\oplus} \mathbf{z}(x, y, t)\right)=\partial_{x} \operatorname{clr}(\mathbf{z}(x, y, t)) ; \quad \operatorname{ilr}\left(\partial_{x}^{\oplus} \mathbf{z}(x, y, t)\right)=\partial_{x} \operatorname{ilr}(\mathbf{z}(x, y, t)) .
$$

Similar expressions hold for $\partial_{y}^{\oplus} \mathbf{z}(x, y, t)$ and $\partial_{t}^{\oplus} \mathbf{z}(x, y, t)$.

Definitions and properties related to integral of simplex-valued functions of one variable have been stated in (Egozcue et al., 2011). They are summarized in the next Proposition.

Proposition 2.2 Let $\mathbf{f}: I \subseteq \mathbb{R} \rightarrow \mathbb{S}^{n}$ be a continuous simplex-valued function of real variable. 
- $A$ differentiable function $\mathbf{F}: I \subseteq \mathbb{R} \rightarrow \mathbb{S}^{n}$ is an antiderivative of $\mathbf{f}$ on $I$ if, and only if, $\partial^{\oplus} \mathbf{F}(\xi)=\mathbf{f}(\xi), \xi \in I$.

- $\int^{\oplus} d \xi \odot \mathbf{f}(\xi)=\mathcal{C} \exp \left(\int \log (\mathbf{f}(\xi)) d \xi\right)$.

- $\int_{[a, b]}^{\oplus} d \xi \odot \mathbf{f}(\xi)=\mathcal{C} \exp \left(\int_{a}^{b} \log (\mathbf{f}(\xi)) d \xi\right)$.

- $\operatorname{clr}\left(\int_{[a, b]}^{\oplus} d \xi \odot \mathbf{f}(\xi)\right)=\int_{a}^{b} \operatorname{clr}(\mathbf{f}(\xi)) d \xi$.

- $\operatorname{ilr}\left(\int_{[a, b]}^{\oplus} d \xi \odot \mathbf{f}(\xi)\right)=\int_{a}^{b} \operatorname{ilr}(\mathbf{f}(\xi)) d \xi$.

Some natural extended definitions and properties can be stated for double and line integrals.

Definition 2.3 (Double integrals) Let $\mathbf{z}: S \times T \subseteq \mathbb{R}^{2} \times \mathbb{R} \rightarrow \mathbb{S}^{n}$ be a STSF. The double integral of $\mathbf{z}$ in $S$ is a composition in $\mathbb{S}^{n}$ given by

$$
\iint_{S}^{\oplus}(d \xi d \eta) \odot \mathbf{z}(\xi, \eta, t)=\mathcal{C} \exp \left(\iint_{S} \log (\mathbf{z}(\xi, \eta, t)) d \xi d \eta\right) .
$$

Proposition 2.3 Properties of double integrals are:

$$
\begin{aligned}
& \operatorname{clr}\left(\iint_{S}^{\oplus}(d \xi d \eta) \odot \mathbf{z}(\xi, \eta, t)\right)=\iint_{S} \operatorname{clr}(\mathbf{z}(\xi, \eta, t)) d \xi d \eta \\
& \operatorname{ilr}\left(\iint_{S}^{\oplus}(d \xi d \eta) \odot \mathbf{z}(\xi, \eta, t)\right)=\iint_{S} \operatorname{ilr}(\mathbf{z}(\xi, \eta, t)) d \xi d \eta
\end{aligned}
$$

Definition 2.4 (Line integrals) Let $\mathbf{z}: S \times T \subseteq \mathbb{R}^{2} \times \mathbb{R} \rightarrow \mathbb{S}^{n}$ be a STSF. The line integral of $\mathbf{z}$ along a regular curve $\Gamma \subset S$ of finite length, is

$$
\int_{\Gamma}^{\oplus} d s \odot \mathbf{z}(x(u), y(u), t)=\mathcal{C} \exp \left(\int_{a}^{b} \log (\mathbf{z}(x(u), y(u), t)) s^{\prime}(u) d u\right),
$$

where $\gamma_{1}=(x(a), y(a)), \gamma_{2}=(x(b), y(b))$ are the end points of the curve $\Gamma$ and $s^{\prime}(u)$ denotes the ordinary derivative of $s$ with respect to the parameter $u$.

Proposition 2.4 Properties of line integrals are:

$$
\begin{aligned}
& \operatorname{clr}\left(\int_{\Gamma}^{\oplus}(d s \odot \mathbf{z}(x(u), y(u), t))\right)=\int_{a}^{b} \operatorname{clr}(\mathbf{z}(x(u), y(u), t)) s^{\prime}(u) d u, \\
& \operatorname{ilr}\left(\int_{\Gamma}^{\oplus}(d s \odot \mathbf{z}(x(u), y(u), t))\right)=\int_{a}^{b} \operatorname{ilr}(\mathbf{z}(x(u), y(u), t)) s^{\prime}(u) d u .
\end{aligned}
$$

\section{$3 \quad$ Simplicial Mass Continuity Equation and Differential Operators}

When $n$ species are mixed in a continuum, a common assumption is that the mass of each species changes according the input-output of it through the border of a fixed control volume $V$. When working in a space of dimension $d=2$, volume means area, or alternatively, for $d=1$ is just a length. Notation $V$ is used both for referring to the volume itself and for indicating its magnitude in 
some volume unit. The continuity of mass for each species is normally described by the continuity equation (Landau and Lifshitz, 1987; White, 1991). It can be written as

$$
\partial_{t} \rho_{k}+\operatorname{div}\left(\rho_{k} \vec{v}_{k}\right)=0 \quad, \quad k=1,2, \ldots n,
$$

where $\rho_{k}$ is the mass density of the $k$-species, and $\vec{v}_{k}=\left(v_{k x}, v_{k y}\right)$ is its velocity in a planar movement. Attention should be paid to the definition of $\rho_{k}$. It is the ratio of the mass $m_{k}$ to the volume $V_{k}$ occupied by the mass of the $k$-species. Accordingly, $\rho_{k}=m_{k} / V_{k}$ and the units can be, for instance, $\mathrm{g} / \mathrm{cm}^{3}$. Interest is centered in the behaviour of (mass) concentration $c_{k}=m_{k} / M$ of each species, which is given as the ratio of the mass of the $k$-species to the total mass $M$ within some given control volume $V$. The overall density is $\rho=M / V$ which leads to a convenient expression of $\rho_{k}$ in terms of concentrations

$$
\rho_{k}=\frac{m_{k}}{V_{k}}=\frac{m_{k} / M}{V_{k} / \rho V}=\rho \frac{m_{k} / M}{V_{k} / V}=\rho \frac{c_{k}}{a_{k}}=\rho d_{k}, k=1,2, \ldots, n,
$$

where $a_{k}=V_{k} / V$ is the volume fraction or volume concentration of the $k$-species. The ratio of mass to volume concentration is denoted $d_{k}=c_{k} / a_{k}$. Note that the continuity equations hold for each species but not for the total, as the change of concentrations modifies the mass content of $V$ and the diffusion or selective transport of some species may change the overall density $\rho$. As a conclusion continuity equation (1) does not hold for the overall density $\rho$ and different velocities $\vec{v}_{k}$. For a planar flow the fields of densities are considered functions of space location $s \in S \subseteq \mathbb{R}^{2}$ and time $t \in T \subseteq \mathbb{R}$. The explicit dependence is suppressed unless it is necessary, for instance, $\rho(s, t)$ is denoted $\rho$.

Substituting in Eq. (1) the expression of $\rho_{k}$ in Eq. (2) and developing the divergence

$$
\partial_{t}\left(\rho d_{k}\right)+\operatorname{div}\left(\rho d_{k} \vec{v}_{k}\right)=\partial_{t}\left(\rho d_{k}\right)+\partial_{x}\left(\rho d_{k} v_{k x}\right)+\partial_{y}\left(\rho d_{k} v_{k y}\right)=0 \quad, \quad k=1,2, \ldots n .
$$

In order to introduce logarithmic derivatives, the equation is divided by $\rho d_{k}$

$$
\frac{\partial_{t}\left(\rho d_{k}\right)}{\rho d_{k}}+\frac{\partial_{x}\left(\rho d_{k} v_{k x}\right)}{\rho d_{k}}+\frac{\partial_{y}\left(\rho d_{k} v_{k y}\right)}{\rho d_{k}}=0 \quad, \quad k=1,2, \ldots n .
$$

This equation is transformed into

$$
\begin{aligned}
\partial_{t} \log \left(\rho d_{k}\right) & =-\left(v_{k x} \partial_{x} \log \left(\rho d_{k}\right)+v_{k y} \partial_{y} \log \left(\rho d_{k}\right)\right)-\left(\partial_{x} v_{k x}+\partial_{y} v_{k y}\right) \\
& =-\left\langle\vec{v}_{k}, \operatorname{grad} \log \left(\rho d_{k}\right)\right\rangle-\operatorname{div}\left(\vec{v}_{k}\right) \\
& =-\partial_{\vec{v}_{k}} \log \left(\rho d_{k}\right)-\operatorname{div}\left(\vec{v}_{k}\right)=0, \quad k=1,2, \ldots, n,
\end{aligned}
$$

where $\langle\cdot, \cdot\rangle$ is the standard Euclidean inner product in $\mathbb{R}^{2}$ and known properties of derivatives of functions of several variables have been applied. Equations (5) for $k=1,2, \ldots, n$ can be placed in an array as

$$
\left(\begin{array}{c}
\partial_{t} \log \left(\rho d_{1}\right) \\
\partial_{t} \log \left(\rho d_{2}\right) \\
\vdots \\
\partial_{t} \log \left(\rho d_{n}\right)
\end{array}\right)=\left(\begin{array}{c}
-D_{\vec{v}_{1}} \log \left(\rho d_{1}\right) \\
-D_{\vec{v}_{1}} \log \left(\rho d_{2}\right) \\
\vdots \\
-D_{\vec{v}_{1}} \log \left(\rho d_{n}\right)
\end{array}\right)+\left(\begin{array}{c}
-\operatorname{div}\left(\vec{v}_{1}\right) \\
-\operatorname{div}\left(\vec{v}_{2}\right) \\
\vdots \\
-\operatorname{div}\left(\vec{v}_{n}\right)
\end{array}\right)
$$

Logarithmic derivatives in left-hand side of Equation (6) are transformed into a simplicial derivative by taking $\operatorname{clr}^{-1}$

$$
\mathcal{C} \exp \left(\begin{array}{c}
\partial_{t} \log \left(\rho d_{1}\right) \\
\partial_{t} \log \left(\rho d_{2}\right) \\
\vdots \\
\partial_{t} \log \left(\rho d_{n}\right)
\end{array}\right)=\mathcal{C} \exp \left(\begin{array}{c}
\partial_{t} \log \rho \\
\partial_{t} \log \rho \\
\vdots \\
\partial_{t} \log \rho
\end{array}\right) \oplus \mathcal{C} \exp \left(\begin{array}{c}
\partial_{t} \log d_{1} \\
\partial_{t} \log d_{2} \\
\vdots \\
\partial_{t} \log d_{n}
\end{array}\right)=\partial_{t}^{\oplus} \mathbf{d}
$$


where $\mathbf{d}$ is a $n$-part composition obtained by the closure of the $\mathbb{R}^{n}$-vector which positive components are the $d_{k}$ 's. Note that a composition with equal components is the neutral element in $\mathbb{S}^{n}$ and this is the reason for cancelling the array containing the terms $\partial_{t} \rho$ in Eq. (7). Vectors in the right-hand side of Eq. (6) need additional definitions and properties. In the standard vector field analysis, the (spatial) gradient and divergence are useful differential linear operators. Previous Eq. (5-6) suggest that similar concepts can be defined for space-time simplicial fields. Definitions and some properties of such operators for $d=2$ follow.

Definition 3.1 (Simplicial (Spatial) Gradient) Let $\mathbf{z}: S \times T \subseteq \mathbb{R}^{2} \times \mathbb{R} \rightarrow \mathbb{S}^{n}$ be a STSF. The simplicial (spatial) gradient is defined as a bivariate STSF, taking values in $\mathbb{S}^{n} \times \mathbb{S}^{n}$, given by

$$
\operatorname{grad}^{\oplus} \mathbf{z}(s, t)=\left(\partial_{x}^{\oplus} \mathbf{z}(s, t), \partial_{y}^{\oplus} \mathbf{z}(s, t)\right) .
$$

Directional derivatives can be expressed as a kind of $\mathbb{R}^{d}$-inner product of the simplicial gradient and the direction in which the directional derivative is taken. However, the fact that simplicial derivatives are in $\mathbb{S}^{n}$ and spatial directions are in $\mathbb{R}^{d}$, introduces notational intricacies.

Proposition 3.1 (Simplicial gradient and directional derivatives) Let $\mathbf{z}: S \times T \subseteq \mathbb{R}^{2} \times$ $\mathbb{R} \rightarrow \mathbb{S}^{n}$ be a STSF and $\vec{u}=\left(u_{x}, u_{y}\right)$ be a vector. Directional derivative and gradient satisfies

$$
\partial_{\vec{u}}^{\oplus} \mathbf{z}(s, t)=\vec{u} \odot \operatorname{grad}^{\oplus} \mathbf{z}(s, t),
$$

where $\odot$ is interpreted as a perturbation-linear combination (Egozcue et al. (2011))

$$
\vec{u} \odot \operatorname{grad}^{\oplus} \mathbf{z}(s, t)=u_{x} \odot \partial_{x}^{\oplus} \mathbf{z}(s, t) \oplus u_{y} \odot \partial_{y}^{\oplus} \mathbf{z}(s, t),
$$

and $\operatorname{grad}^{\oplus} \mathbf{z}$ has been decomposed in their two components $\partial_{x}^{\oplus} \mathbf{z}$ and $\partial_{y}^{\oplus} \mathbf{z}$.

Proof It results from properties of differentiable functions related with directional derivative. $\diamond$.

Definition 3.2 (Simplicial Derivative Along a multiple vector Field) Let $\mathbf{z}: S \times T \subseteq$ $\mathbb{R}^{2} \times \mathbb{R} \rightarrow \mathbb{S}^{n}$ be a STSF with positive components $z_{k}(k=1,2, \ldots, n)$. Let $\mathbf{v}=\left(\vec{v}_{1}, \vec{v}_{2}, \ldots, \vec{v}_{n}\right)$ be a multiple vector field, being $\vec{v}_{k}=\left(v_{k x}, v_{k y}\right), k=1,2, \ldots, n$. The simplicial derivative of $\mathbf{z}$ along the multiple vector field $\mathbf{v}$ is

$$
\partial_{\mathbf{v}}^{\oplus} \mathbf{z}(s, t)=\mathcal{C} \exp \left(\begin{array}{c}
\partial_{\vec{v}_{1}} \log \left(z_{1}(s, t)\right) \\
\partial_{\vec{v}_{2}} \log \left(z_{2}(s, t)\right) \\
\vdots \\
\partial_{\vec{v}_{n}} \log \left(z_{n}(s, t)\right)
\end{array}\right),
$$

where $\partial_{\vec{v}_{k}} \log \left(z_{k}(s, t)\right)=v_{k x} \partial_{x} \log z_{k}+v_{k y} \partial_{y} \log z_{k}$ is an inner product of $\vec{v}_{k}$ and $\operatorname{grad}\left(\log z_{k}\right)$ in $\mathbb{R}^{2}$.

The simplicial derivative along a multiple vector field is not linear in the simplex. A linear combination of compositions like $\left(\alpha_{1} \odot \mathbf{z}_{1}\right) \oplus\left(\alpha_{2} \odot \mathbf{z}_{2}\right)$ is not equal to the perturbation-linear combination of the two derivatives. However, it is linear in the simplex for linear combinations of multiple vector fields.

Definition 3.3 (Simplicial Divergence) Let $\mathbf{z}_{1}, \mathbf{z}_{2}: S \times T \subseteq \mathbb{R}^{2} \times \mathbb{R} \rightarrow \mathbb{S}^{n}$ be two STSFs. The simplicial divergence of the pair $\left(\mathbf{z}_{1}, \mathbf{z}_{2}\right)$ is a composition in $\mathbb{S}^{n}$ given by

$$
\begin{aligned}
\operatorname{div}^{\oplus}\left(\mathbf{z}_{1}, \mathbf{z}_{2}\right) & =\partial_{x}^{\oplus} \mathbf{z}_{1} \oplus \partial_{y}^{\oplus} \mathbf{z}_{2} \\
& =\operatorname{clr}^{-1}\left[\partial_{x} \operatorname{clr}\left(\mathbf{z}_{1}\right)+\partial_{y} \operatorname{clr}\left(\mathbf{z}_{2}\right)\right] .
\end{aligned}
$$


Taking into account previous definitions, and computing $\mathrm{clr}^{-1}$ of the vectors in the right-hand side of Eq. (6)

$$
\mathcal{C} \exp \left(\begin{array}{c}
-\partial_{\vec{v}_{1}} \log \left(\rho d_{1}\right) \\
-\partial_{\vec{v}_{2}} \log \left(\rho d_{2}\right) \\
\vdots \\
-\partial_{\vec{v}_{n}} \log \left(\rho d_{n}\right)
\end{array}\right) \oplus \mathcal{C} \exp \left(\begin{array}{c}
-\operatorname{div}\left(\vec{v}_{1}\right) \\
-\operatorname{div}\left(\vec{v}_{2}\right) \\
\vdots \\
-\operatorname{div}\left(\vec{v}_{n}\right)
\end{array}\right)=\partial_{\mathbf{v}}^{\oplus} \mathbf{d} \oplus \operatorname{div}^{\oplus}\left(\mathbf{w}_{x}, \mathbf{w}_{y}\right)
$$

where $\mathbf{w}_{x}=\operatorname{clr}^{-1}\left(\mathbf{v}_{x}\right), \mathbf{w}_{y}=\operatorname{clr}^{-1}\left(\mathbf{v}_{y}\right)$; moreover, $\mathbf{v}_{x}, \mathbf{v}_{y}$ are $\mathbb{R}^{n}$-vectors grouping the first and second components of $\vec{v}_{k}$ for $k=1,2, \ldots, n$ respectively. Note that $\operatorname{clr}\left(\mathbf{w}_{x}\right)=\mathbf{v}_{x}-\mathbf{v}_{x 0}$, where $\mathbf{v}_{x 0}$ is a constant-component vector which components are the aritmetic mean of the components of $\mathbf{v}_{x}$; and similarly for $\operatorname{clr}\left(\mathbf{w}_{y}\right)$.

Hence, considering Eqs. (7) and (8), the simplicial mass continuity equation can be written as

$$
\partial_{t}^{\oplus} \mathbf{d}=\partial_{\mathbf{v}}^{\oplus} \mathbf{d} \oplus \operatorname{div}^{\oplus}\left(\mathbf{w}_{x}, \mathbf{w}_{y}\right) .
$$

An important feature is that the overall density $\rho$ does not appear in Eq.(9), therefore, this equation is purely compositional. Note that, in general, this is not a linear equation in the simplex due to the non-linearity of $\partial_{\mathbf{v}}^{\oplus} \mathbf{d}$ with respect to $\mathbf{d}$.

Furthermore, if each fluid species is incompressible, that is $\rho_{k}$ is constant, then Eq. (9) reduces to

$$
\operatorname{div}^{\oplus}\left(\mathbf{w}_{x}, \mathbf{w}_{y}\right)=\mathbf{n} \quad, \quad \mathbf{n}=\mathcal{C}(1,1, \ldots, 1)
$$

quite similar to standard continuity equation for an incompresible fluid flow (White, 1991).

In some cases the simplicial fields $\mathbf{z}_{1}$ and $\mathbf{z}_{2}$ can be the two components of a simplicial gradient, that is, there is a simplicial field $\mathbf{w}$ such that $\left(\mathbf{z}_{1}, \mathbf{z}_{2}\right)=\operatorname{grad}^{\oplus} \mathbf{w}=\left(\partial_{x}^{\oplus} \mathbf{w}, \partial_{y}^{\oplus} \mathbf{w}\right)$. In these cases, the bivariate simplicial field $\left(\mathbf{z}_{1}, \mathbf{z}_{2}\right)$ is said to derive from the potential composition $\mathbf{w}$, again following the ideas of the standard vector analysis. An important differential operator in this situation is the Laplacian $\Delta=\partial_{x}^{2}+\partial_{y}^{2}$. The compositional counterpart of the Laplacian can be defined as follows.

Definition 3.4 (SIMPLiCial LAPLACiAn) Let $\vec{x}$ be a location in $R$ and $\mathbf{w}$ be a STSF defined in a neighbourhood of $(\vec{x}, t)=(x, y, t)$. The simplicial Laplacian of $\mathbf{w}$ is a composition in $\mathbb{S}^{n}$ given by

$$
\Delta^{\oplus} \mathbf{w}=\operatorname{div}^{\oplus}\left(\operatorname{grad}^{\oplus} \mathbf{w}\right)=\partial_{x}^{\oplus 2} \mathbf{w} \oplus \partial_{y}^{\oplus 2} \mathbf{w},
$$

where the symbol $\partial^{\oplus 2}$ is the second order simplicial derivative (Egozcue et al., 2011).

To show the relevance of previous definitions, it is worth to state a compositional extension of the Gauss divergence theorem, here stated in $\mathbb{R}^{2}$.

Theorem 3.1 Let $\mathbf{z}_{1}, \mathbf{z}_{2}: S \times T \subseteq \mathbb{R}^{2} \times \mathbb{R} \rightarrow \mathbb{S}^{n}$ be two STSF's, differentiable up to second order. Let $R$ be a bounded and connected domain in the plain with piecewise regular and closed boundary $\Gamma$. Consider $x, y$ as the Cartesian plain coordinates, and a piecewise regular parametrization $x=x(u)$, $y=y(u)$ of the boundary $\Gamma$. Then,

$$
\iint_{R}^{\oplus}(d x d y) \odot \operatorname{div}^{\oplus}\left(\mathbf{z}_{1}(x, y, t), \mathbf{z}_{2}(x, y, t)\right)=\int_{\Gamma}^{\oplus} d s \odot\left(\partial_{\vec{n}_{x}} \mathbf{z}_{1}(x(u), y(u), t) \oplus \partial_{\vec{n}_{y}} \mathbf{z}_{2}(x(u), y(u), t)\right)
$$

where $\vec{n}_{x}=-y^{\prime}(u), \vec{n}_{y}=x^{\prime}(u)$ are, respectively, the vector fields of the first and second components of the normal direction to the boundary $\Gamma$; and $d s=\sqrt{x^{\prime}(u)^{2}+y^{\prime}(u)^{2}} d u$. 


\section{Conclusions}

The study of space-time simplicial fields reveals some interesting aspects and properties for applications in problems related with space-time evolutionary compositions. The present contribution is not complete and needs to be developed further.

It is possible to define, in a natural way, simplicial differential operators, similar to standard equations appearing in fluid mechanics and vector fields in general. The continuity equation of mass in fluid mechanics has been studied in some detail as a motivation to introduce some definitions. However, one of the needed simplicial differential operators, is not linear in the simplex, thus introducing features which are not dealt with in the standard formulation. It seems possible and useful to study the simplicial version of some important equations in Fluid Mechanics and other parts of Physics, such as advection-diffusion, Navier-Stokes and others in their compositional formulation.

\section{Acknowledgements}

This research has been supported by the Spanish Ministry of Education and Science under project 'METRICS' (Ref. MTM2012-33236); and from the Agència de Gestió d'Ajuts Universitaris i de Recerca of the Generalitat de Catalunya under the project Ref. 2009SGR424.

This research has been supported by the Agència de Gestió d'Ajuts Universitaris i de Recerca (AGAUR) of the Generalitat de Catalunya (GENCAT) under the project "Compositional and Spatial Analysis" (COSDA, Ref: 2014SGR551; 2014-2016).

\section{References}

Aitchison, J. (1986). The Statistical Analysis of Compositional Data. Monographs on Statistics and Applied Probability. London (UK): Chapman \& Hall Ltd., London (UK). (Reprinted in 2003 with additional material by The Blackburn Press). 416 p.

Egozcue, J. J., C. Barceló-Vidal, J. A. Martín-Fernández, E. Jarauta-Bragulat, J. L. DíazBarrero, and G. Mateu-Figueras (2011). Elements of simplicial linear algebra and geometry. See Pawlowsky-Glahn and Buccianti (2011).

Egozcue, J. J., E. Jarauta-Bragulat, and J. L. Díaz-Barrero (2011). Calculus of simplex-valued functions. See Pawlowsky-Glahn and Buccianti (2011).

Egozcue, J. J., V. Pawlowsky-Glahn, G. Mateu-Figueras, and C. Barceló-Vidal (2003). Isometric logratio transformations for compositional data analysis. Mathematical Geology 35(3), 279-300.

Landau, L. D. and E. M. Lifshitz (1987). Fluid Mecahnics (2nd ed.), Volume 6 of Course of Theoretical Physics. Pergamon Press, New York. 531pp.

Pawlowsky-Glahn, V. and A. e. Buccianti (Eds.) (2011). Compositional Data Analysis: Theory to Applications. Wiley \& Sons.

White, F. M. (1991). Viscous fluid flow. Mechanical Engineering. McGraw Hill International Editions. 614 pp. 